\title{
Chronic Chlamydial Diseases: From Atherosclerosis to Urogenital Infections
}

\author{
Cory Ann Leonard • Nicole Borel
}

Published online: 27 August 2014

(C) Springer International Publishing AG 2014

\begin{abstract}
Chlamydiae cause a wide range of diseases in human and animal hosts. Chlamydia pneumoniae and Chlamydia trachomatis are important human pathogens with worldwide distribution that produce significant morbidity. Acute infections with $C$. pneumoniae cause respiratory tract infections, while chronic infection has been linked to chronic bronchitis, asthma and atherosclerosis. Ocular serovars of C. trachomatis induce trachoma, the leading cause of infectious blindness worldwide. C. trachomatis is the most common bacterial cause of sexually transmitted diseases (STDs) worldwide. Acute infections with genital serovars of C. trachomatis remain clinically silent in most women, but can progress to upper genital tract infection leading to pelvic inflammatory disease (PID), infertility and ectopic pregnancy. Chlamydia-induced, reactive arthritis can develop as a lateterm condition after genital C. trachomatis or respiratory C. pneumoniae infection. In this review, we address recent information on pathogenesis, immune response, diagnosis and treatment of chronic diseases induced by $C$. trachomatis and C. pneumoniae.
\end{abstract}

Keywords Atherosclerosis · Arthritis · Chlamydia pneumoniae $\cdot$ Chlamydia trachomatis $\cdot$ Pelvic inflammatory disease $\cdot$ Trachoma

\footnotetext{
C. A. Leonard · N. Borel

Department of Pathobiology, Institute of Veterinary Pathology, University of Zurich, Winterthurerstrasse 268, CH-8057 Zurich, Switzerland

N. Borel $(\bowtie)$

Institute of Veterinary Pathology, Vetsuisse Faculty, University of Zurich, Winterthurerstrasse 268, CH-8057 Zurich, Switzerland e-mail: N.Borel@access.uzh.ch
}

\section{Introduction}

The chlamydiae, Gram-negative, obligate intracellular bacteria, cause a broad range of diseases, affecting a wide range of economically important non-human animals and humans. All chlamydiae share a biphasic developmental cycle. The primary developmental forms of chlamydiae are elementary bodies (EB) and reticulate bodies (RB). EB, the extracellular form of the bacteria, are small $(0.2 \mu \mathrm{m})$ and infectious, targeting host mucosal epithelial cells. After attachment and entry into the host cell, EB develop within a membrane-bound endocytic vacuole called an inclusion. Inside the inclusion, EB differentiate into larger $(0.8 \mu \mathrm{m})$, metabolically active but noninfectious $\mathrm{RB}$, which undergo multiple rounds of division. $\mathrm{RB}$ redifferentiate into infectious EB, and mature EB complete the developmental cycle, exiting the host cell via lysis or extrusion of the inclusion [1]. Stressors of developing chlamydiae, including the host immune response, nutrient deprivation, antibiotic exposure or co-infection with viruses or parasites, can result in a form called aberrant bodies (AB). AB develop when $\mathrm{RB}$ replicative division and maturation of $\mathrm{RB}$ to $\mathrm{EB}$ is interrupted, resulting in abnormally large chlamydiae. This divergence from the typical developmental cycle constitutes a viable but noninfectious form of chlamydiae, and is termed persistence or the chlamydial stress response, a reversible condition that can eventually allow continued production of infectious EB [1, 2, 3•, 4].

In humans, chlamydiae usually cause eye, urogenital or respiratory infections. Chlamydia trachomatis is the most common bacterial sexually transmitted disease (STD) and the leading cause of infectious blindness worldwide. Chlamydia pneumoniae, an agent of respiratory infection, is nearly ubiquitous in humans, with seropositivity rates of $70-80 \%$ in older populations, suggesting most people experience infection during their lifetime [5]. Chlamydia psittaci, a common pathogen of birds, has the best known zoonotic potential of the 
human pathogenic chlamydiae and causes relatively rare respiratory infections associated with severe clinical manifestations, while several other animal pathogenic chlamydial species, including Chlamydia abortus, Chlamydia felis, and Chlamydia suis, are known, or suspected, to cause infrequent human infections with various clinical presentations $[6,7]$. Chronic chlamydial infections in animals, particularly ruminants and pigs, are sub-clinical and ubiquitous in nature $[8,9]$. Although their pathogenic significance is debateable, recent data suggest clinical impact when these infections coincide with various epidemiological risk factors. Chronic chlamydial human disease has well-recognized medical significance, encompassing the most detrimental outcomes of chlamydial disease, and is the topic of this review.

By definition, chronic diseases are long-lasting conditions that can be controlled, but not cured. The term chronic, however, is usually applied when the disease lasts more than three months, regardless of the eventual outcome [10]. Chronic disease is the leading cause of death and disability in the United States [11]. In Europe, chronic conditions account for $86 \%$ of all deaths and $77 \%$ of the disease burden [12]. Chronic diseases also significantly increase health care costs related to longterm medical care. Exposure to infectious agents has been implicated as a risk factor for development of chronic diseases, and strong association has been recently shown for five pathogens: human immunodeficiency virus (HIV), hepatitis C virus, Helicobacter pylori, and Chlamydia (C.) pneumoniae [12]. Association of chlamydiae other than C. pneumoniae with chronic disease is also well supported.

In this article, we review the role of chlamydiae in chronic disease conditions including atherosclerosis, trachoma, urogenital infections and arthritis. Chlamydiae have been implicated in neurological chronic diseases, such as multiple sclerosis and Alzheimer's disease, and in neurobehavioral diseases such as autism and schizophrenia [13], but because supporting evidence is contradictory, these diseases will not be discussed here. Additionally, chlamydial infection has been associated with cervical, ovarian, and prostate cancers [14, 15], but again, evidence supporting the association is inconsistent and this topic will not be discussed at length. Because of length limitations, we focus on the intensively investigated chlamydial species C. trachomatis and C. pneumoniae in humans. We will: 1 ) address the clinical significance and public health impact of chronic chlamydial diseases; 2) summarize pathogenesis emphasizing host-pathogen interactions, including host immune response and bacterial factors associated with disease; 3 ) discuss diagnostic methods and therapeutics in the light of chronicity; and 4) indicate future research directions.
Chlamydia trachomatis: Trachoma and Blindness

Trachoma, caused by ocular strains of $C$. trachomatis, causes visual impairment of about 2.2 million people, of whom 1.2 million are irreversibly blind [16]. Globally, it is estimated that more than 50 countries are endemic for blinding trachoma, mainly in Africa and the Middle East, but also in Asia, Latin America and the western Pacific [17]. However, estimates for global trachoma vary considerably due to limited reliable survey data from endemic regions [18]. Trachoma is associated with poor hygiene status and extreme poverty [17]. It is a family-based disease clustering in certain communities and specific households within these communities. The disease is spread by direct contact with ocular and nasal discharges, contact with fomites, or contact with eye-seeking flies, which are vectors for the disease. Trachoma is thus a disease specific to poor rural regions in less developed countries and is part of the Neglected Tropical Diseases Program [16].

Ocular serovars (A, B, Ba, and C) of C. trachomatis have highly specific tropism for mucosal epithelia of ocular conjunctiva. Infection of the conjunctival epithelium leads to conjunctivitis and triggers an immune response characterized by a marked inflammatory cell infiltrate and release of proinflammatory cytokines in the conjunctiva $[18,19 \bullet]$. One episode of infection results in self-limiting chlamydial conjunctivitis, an acute phase referred to as active trachoma. The World Health Organization (WHO) estimates 40 million people worldwide have active trachoma [16]. Infection is usually acquired in infancy in hyperendemic regions and active trachoma is mostly seen in children, progressing to eyelid scarring and blindness in adulthood. Active trachoma is frequently found in the absence of detectable $C$. trachomatis infection, and is clinically represented by papillary and/or follicular inflammation of the tarsal conjunctiva. Repeated and/or persistent infections trigger sustained inflammation and scarring of the upper tarsal conjunctiva. Scarring and fibrosis, in turn, distort the upper eyelid and facilitate inturning of the eyelid (entropion) and eyelashes (trichiasis), causing irritation of the corneal surface and irreversible blindness. The scarring and trichiasis that lead to corneal opacity and sustained pathological tissue reaction to inflammation constitute the second phase of trachoma.

The World Health Organization Simplified Trachoma Grading System divides active trachoma into two often coexisting phenotypes: Trachoma Inflammation Follicular (TF) and Trachoma Inflammation Intense (TI) [18]. Chronicity and progression to inflammatory eye lesions are classified in the WHO system as Trachomatous Scarring (TS), reflected by tarsal conjunctiva scarring, and Trachomatous Trichiasis (TT), including at least one eyelash rubbing on the eyeball. The most severe disease sequela is blinding Corneal Opacity (CO) [18]. The immune response to $C$. trachomatis provides only partial protection, is serovar-specific and does not 
prevent reinfection [19॰]. Tissue damage and scarring result from chronic, pathological immune reactions. Related immunity and immunopathogenesis have been studied in mouse and guinea pig animal models, and data more comparable to human trachoma have been obtained from non-human primate studies. Scarring complications result from complex interactions between infectious burden, local immune response and host genetic polymorphisms related to immune function.

Repeated reinfections are implicated in the development of chronic scarring disease [19•]. The role of persistent, nonreplicating chlamydial forms in ocular infections is unclear and controversial within the chlamydial field [20]. Tissue damage and fibrosis in Chlamydia-related diseases are thought to result from cell-mediated immunity responses against chlamydial antigens, either by delayed type hypersensitivity or molecular mimicry [21]. In trachoma, infected conjunctival epithelial cells secrete pro-inflammatory cytokines, chemokines, and growth factors, which recruit inflammatory immune cells. Inflammatory cells such as neutrophils and macrophages disrupt normal tissue architecture by releasing mediators such as toxic reactive oxygen and nitrogen species and matrix metalloproteinases (MMP). MMP9 can degrade collagen IV, leading to basement membrane disruption, and pro-fibrinogenic factors are thought to stimulate activated fibroblasts to produce collagen, causing scarring.

Key factors influencing trachoma development and progression are the presence of different strains circulating within communities, pathogen burden of infected individuals and polymorphisms in specific host genes. Host polymorphism in immune response genes is hypothesized to play a significant role in trachoma disease progression [22-]. Single nucleotide polymorphisms in the Interleukin-10 (IL-10) gene, the tumor necrosis factor (TNF) locus and MMP-9 have been implicated in trachoma pathogenesis [22•]. Recently, specific combinations of polymorphisms in Human Leucocyte Antigen C (HLA-C) ligands and their inhibitory Killer-cell Immunoglobulin-like Receptors (KIRs) were associated with increased risk of conjunctival scarring in trachoma patients [23]. Besides host polymorphisms, genetic variation of C. trachomatis impacts disease severity and tissue tropism [22•]. Major outer membrane protein (MOMP) serovar predicts chlamydial disease biovars (A-C: endemic trachoma), but does not reflect disease severity differences. Polymorphisms in chlamydial genes such as Tarp, Inc, CT229, pmp and cytotoxin appear to influence disease severity and tissue tropism; however, clear links and mechanisms are unknown. Distinction between ocular and genital strains can also be made based on mutations in the tryptophan synthase genes [24].

Documented C. trachomatis infection correlates poorly with clinical sequelae [25], complicating diagnosis of trachoma. Nucleic acid amplification tests (NAATs) are sensitive and specific, but results do not correlate with clinical grading.
In vivo confocal microscopy has been recently used to visualize progression of inflammatory and scarring changes [26]. Commercial NAATs do not detect Chlamydiaceae species other than C. trachomatis. Single and mixed infections with C. trachomatis, C. psittaci, C. suis, C. pecorum and C. pneumoniae were detected in conjunctival samples of trachoma patients by ArrayTube microarray [27]. This finding and the potential zoonotic origin of these Chlamydiaceae species other than $C$. trachomatis might have implications for immunopathology and disease outcome in trachoma patients, therapeutic treatment and future vaccine development.

The WHO launched an initiative with the ambitious goal of eliminating blinding trachoma globally by 2020 . The SAFE strategy includes: surgery for trichiasis, antibiotics for active trachoma, facial cleanliness, and environmental improvement [16]. Current WHO recommendations constitute mass treatment with a single dose of azithromycin. The risk of adverse events and possible antibiotic resistance development due to azithromycin treatment merit consideration, but ancillary benefits such as reduced infectious disease and decreased childhood mortality outweigh these concerns [25]. Success of trichiasis surgery is impeded by high recurrence rate (5$40 \%$ ), poor surgical technique, limited accessibility to surgery and lack of acceptance of surgery among the local population [28]. Limiting exchange of ocular secretions can be achieved by facial cleanliness and improvement of hygiene conditions (environmental improvement) to decrease transmission [28].

\section{Chlamydia trachomatis: Genital Infections}

C. trachomatis is the most common bacterial cause of STD worldwide. In the United States, 1.4 million chlamydial infections were reported by the Centers for Disease Control and Prevention (CDC) in 2011 [29], and the WHO estimates that more than 90 million persons are infected worldwide [30]. The greatest burden is in sexually active women aged 14 to 19 years, with a prevalence of approximately $6.8 \%$ in the United States [31]. The major age groups for chlamydial STD are women aged 18 to 20 and men aged 20 to 24 years. Moreover, many patients are asymptomatic (70-90\% of women, $30-50 \%$ of men), thus most cases likely remain undiagnosed/unreported [29]. Risk factors include young adulthood, multiple sex partners, intermittent condom use, cervical ectopy, history of other STD such as HIV, low education status, low socioeconomic class, and anal receptive intercourse [29]. High-risk types of human papillomavirus (HPV) are principle causative agents in cervical cancer, but C. trachomatis infection is a co-factor in development of cervical neoplasia [32]. Women with untreated chlamydial diseases have increased risk of HIV infection [33]. Additionally, HIV-infected women with reduced CD4+ T cell counts have increased risk for developing $C$. trachomatis pelvic inflammatory disease (PID) [34•]. Forty-six percent of men 
and women infected with Neisseria gonorrhoeae also have co-infection with C. trachomatis [29].

Nineteen serovars based on MOMP seroreactivity predict chlamydial disease biovars: A-C (endemic trachoma), D-K (genital diseases) and L1-L3 (lymphogranuloma venereum). C. trachomatis genital serovars infect superficial mucosal epithelia of the urethra in men or endocervix in women, initiating disease. In women, $C$. trachomatis cervical infections are mostly asymptomatic and can either resolve spontaneously or progress for weeks to months, causing complications [33]. Approximately $25 \%$ of women with chlamydial cervicitis have concomitant urethritis [33]. Cervical infection can ascend into the endometrium and fallopian tubes and develop into chronic infection and PID. PID is characterized by infection and inflammation of the upper genital tract, frequently involving the endometrium, fallopian tubes, and pelvic peritoneum (endometritis, salpingitis or tubo-ovarian abscess and peritonitis). PID is caused by common sexually transmitted infections, such as Neisseria gonorrhoeae, Mycoplasma genitalium, C. trachomatis (in $30 \%$ of clinical cases), and by anaerobic vaginal microbes causing bacterial vaginosis [35]. Presumptive diagnosis of PID is made clinically (women of reproductive age with pelvic or abdominal pain), while definitive diagnosis is made by laparoscopy [35]. If untreated, $8-10 \%$ of C. trachomatis-infected women develop PID [35]. Tubal damage is mediated by innate immune responses and adaptive T-cell responses. Long-term sequelae of PID include tubal infertility, ectopic pregnancy, and chronic pelvic pain caused by tubal damage and scarring from inflammation. The duration of an infection or repeated infections affect the pathogenesis of PID, but the relative importance if each has not been elucidated. Repeated $C$. trachomatis infections are common, indicating limited natural immunity.

Women with active infection can transmit $C$. trachomatis to their infant during delivery, leading to conjunctivitis and pneumonia in the newborn. C. trachomatis infection in pregnant women has been also linked to chorioamnionitis, placentitis, premature rupture of membranes, and preterm birth [36], however, existing evidence is weak [32]. In men, C. trachomatis causes non-gonococcal urethritis, epididymitis, prostatitis, and proctitis [37]. Urethritis is the most frequent STD syndrome in men, and $C$. trachomatis is the causative agent in 15-40\% of cases. The more invasive strains causing lymphogranuloma venereum (LGV) are named L1, L2, L2a and L3. LGV serovars infect and replicate within macrophages, spread systemically through lymph nodes, and cause necrosis and abscesses in inguinal and femoral lymph nodes. LGV proctitis can vary from clinically silent to severe. Traditionally, LGV is found most often in Africa, India, Southeast Asia, and the Caribbean, and almost exclusively in men who have sex with men (MSM). Since 2004, increasing incidence has been found in North America, Europe, and Australia and occurs mostly in MSM with proctitis [29].
C. trachomatis infection of the female genital tract is recognized by Toll- like receptor (TLR)-2 and TLR-4 and nucleotide-binding oligomerization domain (NOD)1, leading to induction of interferon (IFN) gamma. Cell-mediated immunity is important in clearance of C. trachomatis infection. However, the immune response against $C$. trachomatis infection does not provide long-lasting protection and may contribute to pathology. Th1 T helper cell response helps resolve the infection, but also leads to secretion of pro-inflammatory factors such as TNF alpha, IL-1alpha and IL-6. Induction of IL-10 might down regulate the chlamydial-specific T-cell responses, leading to chronic inflammation and tissue damage in persistent infections [38]. Matrix metalloproteinase 9 (MMP9) expression by fallopian tube cells infected with C. trachomatis is associated with scarring [34•]. Clearance of $C$. trachomatis infection might be delayed by pathogen immune evasion strategies, such as enhanced survival inside and outside host cells, reduced inflammatory and adaptive immune responses and ability to persist within host cells as $\mathrm{AB}$ [39]. Adding complexity, sex hormones modulate female genital tract immune responses [40, 41]. Women are more susceptible to chlamydial infection under the influence of estradiol, and estradiol enhances disease sequelae [40]. Tcell-driven INF gamma and Th17 responses are critical for clearing infection and play a role in protection from disease $[38,42]$. Initiation of autoimmunity by molecular mimicry has been suggested in the pathogenesis of PID [43]. The most important factor in molecular mimicry might be chlamydial heat shock protein 60 (Hsp60). Chlamydial Hsp60 is considered the key antigen in immunopathogenesis of tubal infertility, stimulating humoral and cell mediated immune responses in women with PID/tubal infertility [43]. Increased antibody response to chlamydial Hsp60 in women is strongly associated with PID, ectopic pregnancy, and tubal infertility [44].

Variability in the MOMP gene (ompA) is unrelated to disease severity and MOMP serovars fail to correlate with virulence [45]. However, recent data indicate that variation in the pmp genes may contribute to disease severity [22•]. Pmps $\mathrm{B}, \mathrm{D}$, and $\mathrm{H}$ are strongly immunogenic and elicit proinflammatory cytokine responses [45]. Genital, but not ocular, strains of $C$. trachomatis possess functional tryptophan synthase (trpBA) to convert indole, secreted by local vaginal flora, into tryptophan. A perturbed vaginal microbiome (bacterial vaginosis) might provide a source of indole, enabling genital $C$. trachomatis serovars to circumvent tryptophan limiting bacteriostatic/bactericidal effects of IFN gamma [46] and establish persistent infection [4]. Persistence versus clearance is likely driven by IFN gamma responses. High levels of IFN gamma eradicate chlamydiae, but low levels result in the persistent state [42]. Inflammation of infected tissues promotes local oxygen consumption, resulting in hypoxia [47]. A recent study indicates hypoxia reactivates IFN gamma-induced persistent $C$. trachomatis, causing increased 
bacterial growth and progeny, while dampening the host inflammatory response [48].

The $C$. trachomatis genome is highly conserved, and diversity seems to have evolved through genetic recombination [49] and might result in hypervirulent strains [22•]. Deletion events also occur and have been described in the cryptic plasmid of $C$. trachomatis. Chlamydial plasmids are present in C. trachomatis, but are non-conjugative and nonintegrative; they do not encode antibiotic resistance nor show signs of genetic flexibility. Therefore, they are targeted for NAAT diagnosis of $C$. trachomatis infection [50]. However, emergence of the new Swedish variant (nvCT), a mutated strain from serovar E strains carrying a 377-base-pair deletion within its plasmid, was reported in 2006. Several commercial NAATs targeted this region, leading to diagnostic failure [51]. Retrospective studies suggest nvCT in the Swedish population arose after 2000, perhaps by importation of the variant or by spontaneous mutation [49]. Genetic predisposition and host immune response are also important in pathogenesis of long-term complications of genital C. trachomatis infection [32]. Specific HLA DQ alleles and polymorphisms in the promoter of IL-10 and TNF alpha are associated with high risk of tubal infertility [22•], whereas polymorphisms in TLR-2 are associated with protection against tubal disease following C. trachomatis infection [36].

Diagnosis of $C$. trachomatis infection is recommended by NAAT from urine, vaginal, or endocervical swabs. NAAT from non-genital samples, such as rectal swabs, is performed but not US Food and Drug Administration (FDA)-approved and is essential to diagnose LGV proctitis [33]. Self-collected vaginal or urine swabs are most commonly used in screening programs. The Centers for Disease Control and Prevention (CDC) recommends annual screening for $C$. trachomatis in sexually active women aged 25 years and younger [31]. Upon diagnosis, primary uncomplicated urogenital infections can be effectively treated with antibiotics. The CDC recommends either single-dose azithromycin or a 7-day course of doxycycline in adolescent and adult men and women, and amoxicillin in pregnant women [52]. Early treatment of C. trachomatisinfected individuals shortens infection duration and prevents sexual transmission and complications including PID, while treatment of pregnant women prevents transmission to the infant during delivery [52]. However, most chlamydial infections are asymptomatic and undiagnosed/untreated. Treatment failure occurs [42], perhaps due to re-infection, persistent infection or, less likely, acquired antibiotic resistance. Recurrent $C$. trachomatis infections result from re-infection from an untreated partner or infection from a new partner [52]. Moreover, prevalence of anorectal chlamydia is high; almost all women with anorectal chlamydia had concurrent urogenital chlamydia [53]. This finding has implications for the diagnosis of $C$. trachomatis infections (both sites should be tested) and for the therapy (anorectal chlamydial infections are more difficult to treat). Moreover, anorectal infection might lead to recurrent vaginal infection by autoinoculation [54]. Treatment of LGV infection requires prolonged therapy (21 days) and the CDC recommends doxycycline [52].

Screening programs aim to identify and treat asymptomatic cases of cervicitis before $C$. trachomatis infection can progress to PID [35]. Trials indicate that Chlamydia screening and treatment reduce risk of PID among young women [55]. Screening has contributed to the decline of PID; however, the magnitude of benefit might have been overestimated in initial trials. Asymptomatic chlamydial infections may have been present for months when detected by screening, and thus might have already progressed to chronic inflammation in the upper genital tract. The role of chlamydial screening in reducing complications has not been confirmed and benefit on an individual level is difficult to assess [55]. Future focus should include screening young, sexually active women; determining optimal frequency of screening and benefit of screening for repeat infections; and, of major importance, treatment of partners of infected women [56]. In addition to preventing adverse sequelae of $C$. trachomatis infection, reducing the incidence of new infections through interruption of transmission might be equally important [55]. Despite screening and control programs, reported $C$. trachomatis cases have not exhibited sustained declines. This may be explained by the arrested immunity hypothesis: early treatment interrupts acquisition of protective immunity, increasing risk of reinfection [57, 58]. A recent clinical study provides data supporting this concept, indicating spontaneous resolution of chlamydial infection in the absence of antibiotic treatment may reduce risk of subsequent reinfection [59]. Development of a safe and effective $C$. trachomatis vaccine to prevent acquisition and transmission of infection, and prevent development of inflammatory sequelae, remains the ultimate goal $[42,57]$.

Chlamydia pneumoniae: Respiratory Infections, Coronary Artery Disease and Atherosclerosis

Chlamydia (C.) pneumoniae causes a wide range of acute and chronic respiratory diseases and has been associated with atherosclerosis and cardiovascular disease. The chlamydial organism Taiwan acute respiratory agent (TWAR) was classified in 1989 as a new species, C. pneumoniae [60]. Human C. pneumoniae strains are highly conserved, in contrast to animal strains, which are thought to be ancestral [61]. Although C. pneumoniae is an acute human respiratory pathogen, much research has focused on its role in chronic infections. C. pneumoniae is essentially ubiquitous, with seropositivity of $50 \%$ by the age of 20 years and $70-80 \%$ by $60-70$ years [62]. Most acute infections are asymptomatic or manifest as mild, self-limiting upper respiratory tract infections. However, progression to severe upper respiratory tract infections (pharyngitis, sinusitis, and otitis) and lower respiratory 
tract infections, such as acute and chronic bronchitis, asthma, and pneumonia, occurs $[63,64]$. C. pneumoniae is believed to account for around $10 \%$ of community-acquired pneumonia (CAP), $5 \%$ of pharyngitis, bronchitis and sinusitis [65], and can exacerbate chronic bronchitis and asthma.

CAP can be of bacterial and/or viral etiology [66]. Bacterial CAP is usually caused by Streptococcus pneumoniae; other common pathogens involved are Staphylococcus aureus, Haemophilus influenzae, Enterobacteriaceae, Legionella pneumoniae, Mycoplasma pneumoniae, and Chlamydia pneumoniae. A prospective study of German CAP patients identified C. pneumoniae in respiratory samples in only $0.9 \%$ cases by PCR [67]. In contrast, another study identified C. pneumoniae as the second most common pathogen in CAP patients in North and South America, with a global incidence of $7 \%$ [68]. The incubation period is around 21 days, with transmission by respiratory secretions [64].

Clinically, the causative pathogen of CAP is often unidentified, and commercially available FDA-approved NAATs for C. pneumoniae are not available [69]. Microimmunofluorescence testing (MIF) is the reference standard for serodiagnosis of $C$. pneumoniae infection. However, MIF cannot differentiate acute versus chronic infection, nor discriminate between past and persistent infections [70]. Common treatment for CAP is empiric antibiotic therapy with a beta-lactam in combination with macrolides, respiratory fluoroquinolones or tetracyclines [71]. Specific treatment of C. pneumoniae -induced acute respiratory infection is successful with macrolides, tetracyclines, or respiratory fluoroquinolones [66]. However, the benefit of treatment is difficult to assess, as the majority of the studies have relied on serology for the diagnosis of C. pneumoniae-induced respiratory infections [52]. Serology often does not correlate with pathogen detection by culture or PCR, especially in children, where more than $70 \%$ of culture positive children with CAP are seronegative [69].

In respiratory C. pneumoniae infections, the innate immune response is likely mediated by Toll-like receptor (TLR) -2 and TLR4-induced early cytokine and chemokine production via MyD88 [72•]. NOD and NOD-like receptors might play a role in prolonged activation of host cells. Bronchial epithelial cells are the first line of defense during C. pneumoniae infection, but after initial infection, the pathogen resides in macrophages and neutrophils. C. pneumoniae infection is also associated with asthma, and in particular, asthma exacerbation. C. pneumoniae-specific IgE is prevalent in asthma patients, and occurrence of these species-specific antibodies is associated with disease severity [73]. The most widely accepted association of $C$. pneumoniae respiratory infection with exacerbation of asthma is documented by multiple epidemiologic and clinical studies, some of which investigated whether antibiotic treatment in asthma patients improves outcome.
However, macrolides, quinolones, ketolides and tetracyclines all have immunomodulatory activity independent of their antimicrobial activity, which might influence outcome. To date, the benefit of antibiotics in asthma patients without evidence of acute infection remains controversial [52].

Atherosclerosis, the leading cause of death in industrialized countries, causes coronary artery disease (CAD) and cerebrovascular disease, and is defined as a chronic inflammatory disease of multifactorial etiology. It is characterized by endothelial cell injury, proliferation of vascular cells and ensuing accumulation of inflammatory cells that secrete proinflammatory molecules such as IL-1, IL-6 and TNF alpha, inciting an oxidative stress response [74]. Besides well-known cardiovascular risk factors (hypertension, hyperlipidemia, diabetes, smoking and obesity), several infectious agents such as cytomegalovirus, influenza $\mathrm{A}$ virus, hepatitis $\mathrm{C}$ virus, HIV, Helicobacter pylori, periodontal pathogens, and $C$. pneumoniae have been implicated in pathogenesis of atherosclerosis $[75,76]$. However, C. pneumoniae is the only one of these pathogens that has been repeatedly detected in atherosclerotic lesions and cultivated from atheromatous plaques. Association of C. pneumoniae with atherosclerosis and coronary heart disease is based on both seroepidemiological studies and direct detection of the microorganism in the atheroma by PCR, immunohistochemistry, in situ hybridization, electron microscopy and culture. Animal models have also demonstrated that progression of atherosclerotic lesions is accelerated by $C$. pneumoniae infection $[75,77 \cdot]$. In human patients, after initial respiratory infection, the pathogen can disseminate from the lungs through peripheral blood mononuclear cells, replicating and persisting in vascular tissue cells-further suggesting a role in the pathobiology of atherosclerosis.

Chlamydial lipopolysaccharide (LPS) and chlamydial Hsp60 are important $C$. pneumoniae virulence factors. Molecular mimicry between bacterial and self antigens such as heat shock proteins might contribute to pathogenesis [75]. In response to infection with $C$. pneumoniae, acute phase proteins enhance progression and destabilization of atherosclerotic plaques [76]. C. pneumoniae can induce proliferation of vascular smooth muscle cells (VSMCs) and stimulate production of numerous pro-inflammatory mediators and adhesion molecules in VSMCs and endothelial cells [76, 78, 79]. C. pneumoniae infection can also induce reactive oxygen species (ROS) in macrophages, platelets, endothelial cells and VSMCs, contributing to atherosclerotic lesion development [74]. Progression of atherosclerotic lesions is viewed as host response to noxious stimuli, and C. pneumoniae infection might represent one among many such possible stimuli. Furthermore, it is unclear if $C$. pneumoniae is involved in induction of atherosclerotic lesions, or if it is a mere colonizer and bystander of already injured vascular tissue. Nevertheless, C. pneumoniae may be the only pathogen for which both a 
direct and an indirect contribution to atherosclerosis have been demonstrated. In evidence of this, antigen and/or DNA/RNA of $C$. pneumoniae has been detected in human plaques, but never found in normal vascular tissue; viable organisms have been isolated from plaques; $C$. pneumoniae-related increased pro-inflammatory response has been shown; and atherosclerotic lesions in mouse and rabbit animal models were accelerated by $C$. pneumoniae infection.

The association between infectious agents, in particular C. pneumoniae, and CAD led to clinical trials assessing antibiotic treatment [75]. Cumulatively, these trials demonstrated that anti-chlamydial antibiotic treatment was ineffective in coronary heart disease patients, leading to the assumption that C. pneumoniae is not involved in the pathogenesis of atherosclerosis [77•]. However, several factors were not considered during these trials: treatment was given to patients suffering "end stage disease" with advanced atherosclerotic lesions unlikely to be influenced by antibiotic treatment; other related pathogens such as bacteria and viruses involved in the atherosclerotic process were not considered; a single antibiotic was used although a combination might have been more effective; and, finally, potential persistent forms within atherosclerotic lesions was not considered, though it is known from in vitro studies that persistent chlamydial organisms are refractory to antibiotic treatment $[77 \cdot]$.

\section{Chlamydia-Induced Arthritis}

The spondyloarthritides (SpA) are rheumatoid factor-negative, HLA-B27-associated arthritic diseases characterized by four clinical features: 1) inflammatory back pain, 2) asymmetric peripheral oligoarthritis, usually of the lower limbs, 3) enthesitis, and 4) extra-articular inflammatory symptoms, most commonly uveitis [80]. Five subgroups comprise the SpA, including ankylosing spondylitis, inflammatory bowel disease-associated $\mathrm{SpA}$, psoriasis-associated $\mathrm{SpA}$, reactive arthritis and undifferentiated SpA. Reactive arthritis (ReA) has similar clinical presentation to other $\mathrm{SpA}$, but specifically develops subsequent to bacterial enteric, urogenital, or, less frequently, respiratory infection, typically within 1 to 3 weeks after the triggering infection [81]. In enteric-associated ReA, Yersinia, Salmonella, Shigella, and Campylobacter predominate, while $C$. trachomatis is the most common in urogenitalassociated $\operatorname{ReA}$, and the respiratory pathogen C. pneumoniae is also associated with the disease [82•]. C. trachomatis genital infections and C. pneumoniae infections account for about $50 \%$ and $12 \%$ of all $\mathrm{ReA}$, respectively [82•, 83]. Septic arthritis, also initiated by infection, but not in the $\mathrm{SpA}$ group, is associated with actively infected synovium/synovial fluids, in contrast to ReA in which synovial cultures are typically negative [81]. However, in Chlamydia-induced ReA, bacteria/ bacterial products can be found in affected joint(s) [83]. This indicates that the traditional description of ReA, having affected but non-infected joints, is inaccurate in the case of Chlamydia-induced ReA. The disease instead shares characteristics of both $\mathrm{ReA}$, defined by autoimmune reactioninduced symptoms, and septic arthritis, defined by infectioninduced inflammation [84•]. Here, we use the term Chlamydia-induced arthritis (CiA) in reference to $\mathrm{ReA}$ induced by $C$. trachomatis and/or C. pneumoniae.

In $\mathrm{CiA}$, and other ReA, all associated clinical features are frequently not present and the clinical presentations can be quite variable. The College of Rheumatology general guidelines can be used as diagnostic criteria for ReA, indicating probable or definitive ReA depending on satisfaction of major and/or minor criteria based on clinical presentation, preceding symptomatic enteric or urogenital infection, and laboratory proof or evidence of triggering or persistent synovial infection [81]. Currently, however, no national or international guidelines exist for diagnosis or classification of ReA, and the incidence and prevalence of $\mathrm{ReA}$ and $\mathrm{CiA}$ remain unclear. The Office of Rare Diseases Research of the National Institutes of Health categorizes ReA as rare, affecting less than 200,000 individuals in the United States (USA) [85]. Notably, with up to $80 \%$ of Chlamydia infections, and up to $90 \%$ of C. pneumoniae infections specifically, expected to be asymptomatic $[84 \bullet, 85]$, it is likely $\mathrm{ReA}$ and $\mathrm{CiA}$ are underdiagnosed. A high incidence of genital Chlamydia infections, estimated to be over 3 million annually in the USA and up to 115 million annually worldwide, and reports that approximately $4 \%$ of those with acute genital chlamydial infection will develop $\mathrm{ReA} / \mathrm{CiA}$, have prompted some to calculate that at least 120,000 new cases of C. trachomatisinduced ReA are likely to occur annually in the USA [82•]. The USA and worldwide prevalence of $\mathrm{CiA}$, if such calculations are accurate, might not be considered rare. Additionally, three recent case reports [86-88] of well-documented CiA following $C$. trachomatis LGV L2 proctitis, all in HIVpositive MSM, indicate that the ongoing increased incidence of $C$. trachomatis LGV L2 proctitis in Europe, Australia and North America [29] may be expected to correlate with increased $\mathrm{CiA}$ incidence. This has led some to suggest that screening for $C$. trachomatis LGV L2 in all HIV-positive MSM presenting with acute arthritis may be beneficial even in the absence of proctitis, because infection can be asymptomatic [86]. ReA is usually self-limiting, with spontaneous symptom resolution within 6 months in approximately $50 \%$ to $70 \%$ of cases; however, $30 \%$ to $50 \%$ of patients progress to chronic infection, often with remitting-relapsing disease course [82 ${ }^{-}$. Significant pain, loss of activity, and economic costs are reported for $\mathrm{ReA}$, and are similar to other arthritic diseases, such as members of the $\mathrm{SpA}$ group and rheumatoid arthritis [89].

Host and bacterial factors contributing to $\mathrm{CiA}$ pathogenesis have not been clearly determined. HLA-B27 allele positivity has traditionally been associated with $\mathrm{CiA} / \mathrm{ReA}$ and the other 
SpA groups. Early studies reported that up to $80 \%$ of ReA cases were HLA-B27 positive, while more recent studies indicate that $30 \%$ to $50 \%$ of all ReA cases are associated with the allele. Thus, HLA-B27 is currently thought to more accurately represent a risk factor for progression to chronic disease and/or increased disease severity in ReA, rather than a risk factor for ReA susceptibility [85]. In ReA other than CiA, the triggering organism is absent from affected joints, and pathogenesis is thought to occur largely based on autoimmune factors initiated by distal or resolved infection. However, viable $C$. trachomatis and/or C. pneumoniae have been demonstrated in affected joints by both electron microscopy and PCR [85]. This suggests that, although host autoimmunity might contribute to pathogenicity, the immune response to an ongoing joint infection may also affect disease pathogenesis.

Viable Chlamydia exists in the joint, apparently as a longterm infection, in a state usually referred to as persistence [85]. Persistent organisms are metabolically active, non-cultivable and exhibit altered patterns of gene expression. Persistence can be induced in vitro and in animal models by a variety of conditions, including amino acid or iron deprivation, antibiotic or IFN gamma exposure, co-infection with viruses or parasites, and heat shock [3•]. Chlamydia disseminates to joints from the initial site of infection via monocytes, and once in the joints, the chlamydiae infect macrophages and/or macrophage-like and fibroblast-like cells of the synovium $[82 \bullet, 84 \bullet, 90]$. Interestingly, the persistent state appears to occur, at least in part, due to Chlamydia infection of monocytes and macrophages, which do not support normal, productive infection [82•]. Cells of the synovium, along with $\mathrm{T}$ cells present in infected joint(s), mount a protective immune response to Chlamydia involving production of cytokines such as IFN gamma, TNF alpha, and various interleukins - a response that is important for clearance of Chlamydia infection, but also involved in inflammatory joint damage via cartilage and bone deterioration [90]. Chlamydial Hsp60 proteins play a major role in the host inflammatory response. In vitro, chlamydial Hsp60 genes show altered expression during persistent infection, and synovial samples from patients with chronic CiA reflect this [82•]. Diagnosis of continued bacterial presence indicates pathogen survival, and thus implies that the immune response is ineffective in clearing the infection. However, this immune response also causes inflammatory joint damage. It has been postulated that damage results from skewing of the $\mathrm{T}$ helper cell response from a Th1 dominant response to a Th2 dominant response, a process in which the persistent state of Chlamydia itself might play a role [84•]. A recent study showed that bacterial DNA and inflammatory cytokine/chemokine and Hsp60 transcripts can be found in the joint during the remitting phase of chronic $\mathrm{CiA}$, suggesting damaging inflammation is ongoing even during symptom remission [91].
Host genetic variability, outside of HLA-B27 phenotype, is likely important in initial and continued immune response to infection, especially considering the low proportion of cases of acute Chlamydia infection resulting in CiA. Initially high levels of IFN gamma and TNF alpha in the synovium are associated with resistance to experimentally induced $\mathrm{CiA}$ [84. ]; and it has subsequently been suggested that hostdetermined low initial cytokine levels at the time of acute Chlamydia infection might potentiate chronic disease progression, perhaps dependent to some degree on the persistent Chlamydia state [92]. Indirect evidence supports the concept that the Chlamydia persistent state may contribute to pathogenesis of CiA. A study evaluating 36 patients with confirmed CiA, all with synovial samples positive for $C$. trachomatis by PCR, exclusively identified ocular, not genital, strains of C. trachomatis [93]. This lead the authors to surmise C. trachomatis ocular strains might have a particular capacity for dissemination from the genital tract, and the small percentage of $C$. trachomatis cases progressing to CiA might reflect the small percentage of cases in which ocular strains are found in genital infection [93]. C. trachomatis ocular strains and C. pneumoniae human strains, the chlamydiae commonly demonstrated to be involved in CiA, are unable to synthesize tryptophan and thus have increased sensitivity to the host IFN gamma response and subsequent tryptophan deprivation, a characteristic that might facilitate persistence as a means of initiating and maintaining chronic infection [94, 95].

Treatment of CiA is not well studied, and therapy relies largely on managing the disease and treating symptoms. Nonsteroidal anti-inflammatory drugs, corticosteroids and the disease modifying anti-rheumatic drug sulfasalazine have shown some efficacy for certain stages or severity of CiA [82•]. A meta-analysis of 12 antibiotic treatment studies showed that long-term antibiotic treatment does not significantly improve disease outcome [96], a finding that may support the concept that continued infection in CiA correlates with, but does not cause, ReA. However, a single trial examining combined 6month use of a macrolide or tetracycline with rifampin, demonstrated that chronic CiA patients showed significant improvement and increased negative Chlamydia PCR diagnosis with combination antibiotic therapy compared to placebo, a finding the authors suggested might reflect that persistent chlamydiae are refractory to standard antibiotic treatment [85]. Anti-TNF alpha treatment, commonly used for rheumatoid arthritis, has shown efficacy for CiA [92]. However, given the importance of TNF alpha and other inflammatory cytokines in control of Chlamydia infection, more studies are needed to evaluate this therapy in CiA. In fact, many confounding factors encompass ReA and CiA, complicating pursuit of effective therapy. The relationship between continued joint infection, subsequent to initial Chlamydia infection, and $\mathrm{CiA}$ disease inception or progression has not yet been proven a causal relationship, and the importance of eradicating 
underlying chlamydial infection for resolution of $\mathrm{CiA}$ is thus unknown. The basic biology underlying the persistent state of Chlamydia in joint infection is also poorly understood, and the in vivo diagnosis, as well as the consequence of persistent infection, remains to be elucidated [20].

\section{Conclusions}

Chronic chlamydial infections are implicated in trachoma, sexually transmitted genital diseases, atherosclerosis and arthritis. These infections are characterized by prolonged and chronic inflammation, scarring and fibrosis mediated by continuous stimulation of the host immune response. Multiple host and chlamydial factors contribute significantly to specific chlamydial chronic diseases. However, several characteristics of chlamydiae and chlamydial infection are especially relevant to the propensity of this organism to establish chronic disease in general: i) infection of multiple cell types and dissemination within the host, ii) the intracellular niche of the chlamydiae, iii) immune evasion allowing enhanced chlamydial survival by modulation of apoptosis, pathogen detection, and inflammatory and adaptive immune responses in the host $[39,97]$, and iv) frequent asymptomatic infection which can make detecting chlamydiae difficult.

Additionally, recent findings have broad implications for diagnosis and treatment of chlamydial infections. First, although the importance of chlamydial persistence in vivo remains unclear, persistent growth forms $(\mathrm{AB})$ can be found in the human endocervix (by electron microscopy) where the local microenvironment at infection is similar to the in vitro model of IFN gamma persistence induction [98]. If the chlamydial persistent state is, in fact, present in vivo, both detection by culture (persistent chlamydiae are by definition noncultivable) and treatment of chlamydial infection may be impacted. Persistent chlamydiae are resistant to killing by antibiotics in vitro; and a model of amoxicillin induced C. muridarum persistence in mice indicates persistent chlamydiae are resistant to killing by azithromycin in vivo as well [99]. Furthermore, clinically relevant concentrations of commonly used penicillins induce $C$. trachomatis persistence in vitro [100]. Second, tetracycline resistance in chlamydiae may be possible. Evidence of antibiotic resistance in human pathogenic chlamydiae is lacking, based on the observation that suspected tetracycline resistant strains lost the resistant phenotype during culture and failed to exhibit genomic evidence of resistance [101]. However, isolation of tetracycline resistant $C$. suis from pigs demonstrates an adaptive ability of chlamydiae to acquire antibiotic resistance under selective pressure [102], with implications for continued use of antibiotic treatment of chlamydial disease. Third, and finally, chlamydiae have long been known to colonize the gastrointestinal (GI) tracts of animal hosts, including poultry and sheep, without causing disease [54]. And although humans can become rectally infected with $C$. trachomatis, long-term intestinal infection in humans has not yet been confirmed [54]. Mouse model studies show chlamydiae infecting the GI tract can persist for up to 100 days with no pathology, and azithromycin treatment sufficient to cure genital infection did not similarly cure GI tract infection, despite drug levels in both anatomical sites [103•]. Thus, intestinal carriage of chlamydiae may not only allow auto- or re-inoculation of the genital tract, but may represent commensal association with the human host, providing a degree of incidental antibiotic resistance [54].

These findings indicate that future research in the areas of chlamydial persistence, mechanisms of protective immunity and immunopathology, and vaccine development is a priority. Given difficulties surrounding diagnosis and antibiotic treatment of chlamydial disease, development of an effective Chlamydia vaccine appears particularly advantageous in the context of chronic disease. Recent data from a non-human primate animal model demonstrated the efficacy of plasmid-deficient chlamydial strains as live attenuated vaccines against genital and ocular chlamydial infections [50], and vaccine development is underway [34•, 42]. Continued study of the intricate biology of the chlamydiae will facilitate advances in prevention, diagnosis and treatment of chronic chlamydial disease.

Acknowledgments The authors would like to thank Dr. Robert Schoborg for critical review of the manuscript.

\section{Compliance with Ethics Guidelines}

Conflict of Interest Cory Ann Leonard and Nicole Borel each declare that there are no conflicts of interest.

Human and Animal Rights and Informed Consent This article contains no studies with human or animal subjects performed by any of the authors.

\section{References}

Papers of particular interest, published recently, have been highlighted as:

- Of importance

1. Abdelrahman YM, Belland RJ. The chlamydial developmental cycle. FEMS Microbiol Rev. 2005;29:949-59.

2. Hogan RJ, Mathews SA, Mukhopadhyay S, Summersgill JT, Timms P. Chlamydial persistence: beyond the biphasic paradigm. Infect Immun. 2004;72:1843-55.

3. Schoborg RV. Chlamydia persistence - a tool to dissect chlamydia - host interactions. Microbes Infect. 2011;13:649-62. Reviews Chlamydia/host interactions, focusing on recent persistence models and detailing the important differences between human and murine immune responses to chlamydial infection. 
4. Wyrick PB. Chlamydia trachomatis persistence in vitro: an overview. J Infect Dis. 2010;201(Suppl):S88-95.

5. Choroszy-Król I, Frej-Mądrzak M, Hober M, Sarowska J, JamaKmiecik A. Infections caused by Chlamydophila pneumoniae. Adv Clin Exp Med. 2014;23:123-6.

6. De Puysseleyr K, De Puysseleyr L, Geldhof J, Cox E, Vanrompay D. Development and validation of a real-time PCR for Chlamydia suis diagnosis in swine and humans. PLoS ONE. 2014;9:e96704.

7. Rohde G, Straube E, Essig A, Reinhold P, Sachse K. Chlamydial zoonoses. Dtsch Arztebl Int. 2010;107:174-80.

8. Reinhold P, Sachse K, Kaltenboeck B. Chlamydiaceae in cattle: commensals, trigger organisms, or pathogens? Vet J. 2011;189:257-67.

9. Schautteet K, Vanrompay D. Chlamydiaceae infections in pig. Vet Res. 2011;42:29.

10. WHO $\mid$ Noncommunicable diseases. World Health Organization; [cited 2014 Aug 12]; Available from: http://www.who.int/topics/ noncommunicable diseases/en/

11. CDC - Chronic Disease - Overview. [cited 2014 Aug 12]; Available from: http://www.cdc.gov/chronicdisease/overview/

12. Orrskog S, Medin E, Tsolova S, Semenza JC. Causal inference regarding infectious aetiology of chronic conditions: a systematic review. PLoS ONE. 2013;8:e68861.

13. De Chiara G, Marcocci ME, Sgarbanti R, Civitelli L, Ripoli C, Piacentini R, et al. Infectious agents and neurodegeneration. Mol Neurobiol. 2012;46:614-38.

14. Chumduri C, Gurumurthy RK, Zadora PK, Mi Y, Meyer TF. Chlamydia infection promotes host DNA damage and proliferation but impairs the DNA damage response. Cell Host Microbe. 2013;13:746-58.

15. Caini S, Gandini S, Dudas M, Bremer V, Severi E, Gherasim A. Sexually transmitted infections and prostate cancer risk: A systematic review and meta-analysis. Cancer Epidemiol. 2014;38: 329-38.

16. WHO | Trachoma. World Health Organization; [cited 2014 Aug 12]; Available from: http://www.who.int/topics/trachoma/en/

17. Wright HR, Turner A, Taylor HR. Trachoma. Lancet. 2008;371: 1945-54.

18. Burton MJ, Mabey DCW. The global burden of trachoma: a review. PLoS Negl Trop Dis. 2009;3:e460.

19. Hu VH, Holland MJ, Burton MJ. Trachoma: protective and pathogenic ocular immune responses to Chlamydia trachomatis. PLoS Negl Trop Dis. 2013;7:e2020. This review gives a comprehensive description of trachoma pathogenesis. It reviews the most recent literature on immunity and immunopathogenesis.

20. Bavoil PM. What's in a word: the use, misuse, and abuse of the word "persistence" in Chlamydia biology. Front Cell Infect Microbiol. 2014;4:27.

21. Stephens RS. The cellular paradigm of chlamydial pathogenesis. Trends Microbiol. 2003;11:44-51.

22. Abdelsamed H, Peters J, Byrne GI. Genetic variation in Chlamydia trachomatis and their hosts: impact on disease severity and tissue tropism. Future Microbiol. 2013;8:1129-46. Comprehensively reviews genetic variation in Chlamydia trachomatis and its impact on disease severity and tissue tropism.

23. Roberts CH, Molina S, Makalo P, Joof H, Harding-Esch EM, Burr $\mathrm{SE}$, et al. Conjunctival scarring in trachoma is associated with the HLA-C ligand of KIR and is exacerbated by heterozygosity at KIR2DL2/KIR2DL3. PLoS Neg1 Trop Dis. 2014;8:e2744.

24. Caldwell HD, Wood H, Crane D, Bailey R, Jones RB, Mabey D, et al. Polymorphisms in Chlamydia trachomatis tryptophan synthase genes differentiate between genital and ocular isolates. J Clin Investig. 2003;111:1757-69.

25. Bhosai SJ, Bailey RL, Gaynor BD, Lietman TM. Trachoma: an update on prevention, diagnosis, and treatment. Curr Opin Ophthalmol. 2012;23:288-95.
26. Hu VH, Massae P, Weiss HA, Cree IA, Courtright P, Mabey $\mathrm{DCW}$, et al. In vivo confocal microscopy of trachoma in relation to normal tarsal conjunctiva. Ophthalmology. 2011;118:747-54.

27. Dean D, Rothschild J, Ruettger A, Kandel RP, Sachse K. Zoonotic Chlamydiaceae species associated with trachoma, Nepal. Emerg Infect Dis. 2013;19:1948-55.

28. Mathew AA, Turner A, Taylor HR. Strategies to control trachoma. Drugs. 2009;69:953-70.

29. Markle W, Conti T, Kad M. Sexually transmitted diseases. Prim Care. 2013;40:557-87.

30. WHO | Sexually transmitted infections (STIs). World Health Organization; [cited 2014 Aug 12]; Available from: http://www. who.int/mediacentre/factsheets/fs110/en/

31. Chlamydia - STD information from CDC. [cited 2014 Aug 12]; Available from: http://www.cdc.gov/std/chlamydia/

32. Paavonen J. Chlamydia trachomatis infections of the female genital tract: state of the art. Ann Med. 2012;44:18-28.

33. Singh D, Marrazzo JM. Screening and management of genital chlamydial infections. Infect Dis Clin North Am. 2013;27:739 53.

34. Rey-Ladino J, Ross AG, Cripps AW. Immunity, immunopathology, and human vaccine development against sexually transmitted Chlamydia trachomatis. Hum. Vaccin. Immunother. 2014;10. Reviews the current knowledge on immunity, immunopathology and vaccine development in sexually transmitted Chlamydia trachomatis infections.

35. Mitchell C, Prabhu M. Pelvic inflammatory disease: current concepts in pathogenesis, diagnosis and treatment. Infect Dis Clin North Am. 2013;27:793-809.

36. Darville T. Recognition and treatment of chlamydial infections from birth to adolescence. Adv Exp Med Biol. 2013;764:109-22.

37. Cunningham KA, Beagley KW. Male genital tract chlamydial infection: implications for pathology and infertility. Biol Reprod. 2008;79:180-9.

38. Darville T, Hiltke TJ. Pathogenesis of genital tract disease due to Chlamydia trachomatis. J Infect Dis. 2010;201(Suppl):S114-25.

39. Brunham RC, Rey-Ladino J. Immunology of Chlamydia infection: implications for a Chlamydia trachomatis vaccine. Nat Rev Immunol. 2005;5:149-61.

40. Hafner LM, Cunningham K, Beagley KW. Ovarian steroid hormones: effects on immune responses and Chlamydia trachomatis infections of the female genital tract. Mucosal Immunol. 2013;6: 859-75.

41. Hall JV, Schell M, Dessus-Babus S, Moore CG, Whittimore JD, Sal M, et al. The multifaceted role of oestrogen in enhancing Chlamydia trachomatis infection in polarized human endometrial epithelial cells. Cell Microbiol. 2011;13:1183-99.

42. Hafner LM, Wilson DP, Timms P. Development status and future prospects for a vaccine against Chlamydia trachomatis infection. Vaccine. 2014;32:1563-71.

43. Hafner L, Beagley K, Timms P. Chlamydia trachomatis infection: host immune responses and potential vaccines. Mucosal Immunol. 2008;1:116-30.

44. Stephens AJ, Aubuchon M, Schust DJ. Antichlamydial antibodies, human fertility, and pregnancy wastage. Infect Dis Obstet Gynecol. 2011;2011:525182.

45. Byrne GI. Chlamydia trachomatis strains and virulence: rethinking links to infection prevalence and disease severity. J Infect Dis. 2010;201(Suppl):S126-33.

46. Aiyar A, Quayle AJ, Buckner LR, Sherchand SP, Chang TL, Zea $\mathrm{AH}$, et al. Influence of the tryptophan-indole-IFN $\gamma$ axis on human genital Chlamydia trachomatis infection: role of vaginal co-infections. Front Cell Infect Microbiol. 2014;4:72.

47. Dietz I, Jerchel S, Szaszák M, Shima K, Rupp J. When oxygen runs short: the microenvironment drives host-pathogen interactions. Microbes Infect. 2012;14:311-6. 
48. Jerchel S, Kaufhold I, Schuchardt L, Shima K, Rupp J. Host immune responses after hypoxic reactivation of IFN- $\gamma$ induced persistent Chlamydia trachomatis infection. Front Cell Infect Microbiol. 2014;4:43.

49. Clarke IN. Evolution of Chlamydia trachomatis. Ann N Y Acad Sci. 2011;1230:E11-8.

50. Rockey DD. Unraveling the basic biology and clinical significance of the chlamydial plasmid. J Exp Med. 2011;208: 2159-62.

51. Unemo M, Seth-Smith HMB, Cutcliffe LT, Skilton RJ, Barlow D, Goulding D, et al. The Swedish new variant of Chlamydia trachomatis: genome sequence, morphology, cell tropism and phenotypic characterization. Microbiology. 2010;156:1394-404.

52. Hammerschlag MR, Kohlhoff SA. Treatment of chlamydial infections. Expert Opin Pharmacother. 2012;13:545-52.

53. Van Liere GA, Hoebe CJ, Wolffs PF, Dukers-Muijrers NH. High co-occurrence of anorectal chlamydia with urogenital chlamydia in women visiting an STI clinic revealed by routine universal testing in an observational study; a recommendation towards a better anorectal chlamydia control in women. BMC Infect Dis. 2014; $14: 274$

54. Rank RG, Yeruva L. Hidden in plain sight: chlamydial gastrointestinal infection and its relevance to persistence in human genital infection. Infect Immun. 2014;82:1362-71.

55. Gottlieb SL, Berman SM, Low N. Screening and treatment to prevent sequelae in women with Chlamydia trachomatis genital infection: how much do we know? J Infect Dis. 2010;201(Suppl): S156-67.

56. Gottlieb SL, Xu F, Brunham RC. Screening and treating Chlamydia trachomatis genital infection to prevent pelvic inflammatory disease: interpretation of findings from randomized controlled trials. Sex Transm Dis. 2013;40:97-102.

57. Brunham RC, Rappuoli R. Chlamydia trachomatis control requires a vaccine. Vaccine. 2013;31:1892-7.

58. Vickers DM, Osgood ND. The arrested immunity hypothesis in an immunoepidemiological model of Chlamydia transmission. Theor Popul Biol. 2014;93:52-62.

59. Geisler WM, Lensing SY, Press CG, Hook EW. Spontaneous resolution of genital Chlamydia trachomatis infection in women and protection from reinfection. J Infect Dis. 2013;207:1850-6.

60. Grayston JT, Kuo C-C, Campbell LA, Wang S-P. Chlamydia pneumoniae sp. nov. for Chlamydia sp. strain TWAR. Int J Syst Bacteriol. 1989;39:88-90.

61. Roulis E, Polkinghorne A, Timms P. Chlamydia pneumoniae: modern insights into an ancient pathogen. Trends Microbiol. 2013;21:120-8.

62. Kuo CC, Jackson LA, Campbell LA, Grayston JT. Chlamydia pneumoniae (TWAR). Clin Microbiol Rev. 1995;8:451-61.

63. Hahn DL, Azenabor AA, Beatty WL, Byrne GI. Chlamydia pneumoniae as a respiratory pathogen. Front Biosci. 2002;7: e66-76.

64. Burillo A, Bouza E. Chlamydophila pneumoniae. Infect Dis Clin North Am. 2010;24:61-71.

65. Blasi F, Tarsia P, Aliberti S. Chlamydophila pneumoniae. Clin Microbiol Infect. 2009;15:29-35.

66. Marrie TJ, Costain N, La Scola B, Patrick W, Forgie S, Xu Z, et al. The role of atypical pathogens in community-acquired pneumonia. Semin Respir Crit Care Med. 2012;33:244-56.

67. Wellinghausen N, Straube E, Freidank H, von Baum H, Marre R, Essig A. Low prevalence of Chlamydia pneumoniae in adults with community-acquired pneumonia. Int J Med Microbiol. 2006;296: 485-91.

68. Arnold FW, Summersgill JT, Lajoie AS, Peyrani P, Marrie TJ, Rossi P, et al. A worldwide perspective of atypical pathogens in community-acquired pneumonia. Am J Respir Crit Care Med. 2007;175:1086-93.
69. Kumar S, Hammerschlag MR. Acute respiratory infection due to Chlamydia pneumoniae: current status of diagnostic methods. Clin Infect Dis. 2007;44:568-76.

70. Villegas E, Sorlózano A, Gutiérrez J. Serological diagnosis of Chlamydia pneumoniae infection: limitations and perspectives. $\mathrm{J}$ Med Microbiol. 2010;59:1267-74.

71. Amin AN, Cerceo EA, Deitelzweig SB, Pile JC, Rosenberg DJ, Sherman BM. The hospitalist perspective on treatment of community-acquired bacterial pneumonia. Postgrad Med. 2014;126:18-29.

72. Shimada K, Crother TR, Arditi M. Innate immune responses to Chlamydia pneumoniae infection: role of TLRs, NLRs, and the inflammasome. Microbes Infect. 2012;14:1301-7. Focuses on the recent progress in understanding Chlamydia pneumoniae pathogenesis and the mechanisms of innate immune response to $C$. pneumoniae infection.

73. Hahn DL, Schure A, Patel K, Childs T, Drizik E, Webley W. Chlamydia pneumoniae-specific IgE is prevalent in asthma and is associated with disease severity. PLoS ONE. 2012;7:e35945.

74. Di Pietro M, Filardo S, De Santis F, Sessa R. Chlamydia pneumoniae infection in atherosclerotic lesion development through oxidative stress: a brief overview. Int J Mol Sci. 2013;14:15105-20.

75. Rosenfeld ME, Campbell LA. Pathogens and atherosclerosis: update on the potential contribution of multiple infectious organisms to the pathogenesis of atherosclerosis. Thromb Haemost. 2011;106:858-67.

76. Tufano A, Di Capua M, Coppola A, Conca P, Cimino E, Cerbone $\mathrm{AM}$, et al. The infectious burden in atherothrombosis. Semin Thromb Hemost. 2012;38:515-23.

77. Campbell LA, Rosenfeld ME, Persistent C. pneumoniae infection in atherosclerotic lesions: rethinking the clinical trials. Front Cell Infect Microbiol. 2014;4:34. Opinion article critically analyzes the negative outcomes in antibiotic trials in patients with atherosclerosis.

78. Kern JM, Maass V, Maass M. Molecular pathogenesis of chronic Chlamydia pneumoniae infection: a brief overview. Clin Microbiol Infect. 2009;15:36-41.

79. Kern JM, Maass V, Maass M. Chlamydia pneumoniae-induced pathological signaling in the vasculature. FEMS Immunol Med Microbiol. 2009;55:131-9.

80. Baraliakos X, Braun J. Spondyloarthritides. Best Pract Res Clin Rheumatol. 2011;25:825-42.

81. Selmi C, Gershwin ME. Diagnosis and classification of reactive arthritis. Autoimmun Rev. 2014;13:546-9.

82. Carter JD, Gerard HC, Whittum-Hudson JA, Hudson AP. The molecular basis for disease phenotype in chronic Chlamydiainduced arthritis. Int J Clin Rheumtol. 2012;7:627-40. Discusses the influence of chlamydial molecular genetic characteristics on pathogenesis in Chlamydia-induced arthritis (CiA), the role of ocular strains of Chlamydia trachomatis in arthritogenesis, and the molecular genetic basis of remitting-relapsing CiA.

83. Zeidler H, Hudson AP. New insights into Chlamydia and arthritis. Promise of a cure? Ann Rheum Dis. 2014;73:63744.

84. Gracey E, Inman RD. Chlamydia-induced ReA: immune imbalances and persistent pathogens. Nat. Rev. Rheumatol. 2012;8:559. Succinctly describes the relationship between spondyloarthritis, reactive arthritis and septic arthritis, and highlights the host immune response to chlamydial infection with emphasis on innate immunity.

85. Carter JD, Inman RD. Chlamydia-induced reactive arthritis: hidden in plain sight? Best Pract Res Clin Rheumatol. 2011;25:359-74.

86. El Karoui K, Méchaï F, Ribadeau-Dumas F, Viard J-P, Lecuit M, de Barbeyrac B, et al. Reactive arthritis associated with L2b lymphogranuloma venereum proctitis. Sex Transm Infect. 2009;85:180-1. 
87. Kober C, Richardson D, Bell C, Walker-Bone K. Acute seronegative polyarthritis associated with lymphogranuloma venereum infection in a patient with prevalent HIV infection. Int J STD AIDS. 2011;22:59-60.

88. Pendle S, Gowers A. Reactive arthritis associated with proctitis due to Chlamydia trachomatis serovar L2b. Sex Transm Dis. 2012;39:79-80.

89. Soderlin MK, Kautiainen H, Skogh T, Leirisalo-Repo M. Quality of life and economic burden of illness in very early arthritis. A population based study in southern Sweden. J Rheumatol. 2004;31:1717-22.

90. Gaston JS. Immunological basis of Chlamydia induced reactive arthritis. Sex Transm Infect. 2000;76:156-61.

91. Gérard HC, Carter JD, Hudson AP. Chlamydia trachomatis is present and metabolically active during the remitting phase in synovial tissues from patients with chronic Chlamydia-induced reactive arthritis. Am J Med Sci. 2013;346:22-5.

92. Carter JD, Hudson AP. The evolving story of Chlamydia-induced reactive arthritis. Curr Opin Rheumatol. 2010;22:424-30.

93. Gerard HC, Stanich JA, Whittum-Hudson JA, Schumacher HR, Carter JD, Hudson AP. Patients with Chlamydia-associated arthritis have ocular (trachoma), not genital, serovars of C. trachomatis in synovial tissue. Microb Pathog. 2010;48:62-8.

94. Huston WM, Barker CJ, Chacko A, Timms P. Evolution to a chronic disease niche correlates with increased sensitivity to tryptophan availability for the obligate intracellular bacterium Chlamydia pneumoniae. J Bacteriol. 2014;196:1915-24.

95. Bonner CA, Byrne GI, Jensen RA. Chlamydia exploit the mammalian tryptophan-depletion defense strategy as a counterdefensive cue to trigger a survival state of persistence. Front Cell Infect Microbiol. 2014;4:17.
96. Barber CE, Kim J, Inman RD, Esdaile JM, James MT. Antibiotics for treatment of reactive arthritis: a systematic review and metaanalysis. J Rheumatol. 2013;40:916-28.

97. Roan NR, Starnbach MN. Immune-mediated control of Chlamydia infection. Cell Microbiol. 2008;10:9-19.

98. Lewis ME, Belland RJ, AbdelRahman YM, Beatty WL, Aiyar AA, Zea AH, et al. Morphologic and molecular evaluation of Chlamydia trachomatis growth in human endocervix reveals distinct growth patterns. Front Cell Infect Microbiol. 2014;4:71.

99. Phillips-Campbell R, Kintner J, Schoborg RV. Induction of the Chlamydia muridarum stress/persistence response increases azithromycin treatment failure in a murine model of infection. Antimicrob Agents Chemother. 2014;58:1782-4.

100. Kintner J, Lajoie D, Hall J, Whittimore J, Schoborg RV. Commonly prescribed $\beta$-lactam antibiotics induce $\mathrm{C}$. trachomatis persistence/stress in culture at physiologically relevant concentrations. Front Cell Infect Microbiol. 2014;4:44.

101. O’Neill CE, Seth-Smith HMB, Van Der Pol B, Harris SR, Thomson NR, Cutcliffe LT, et al. Chlamydia trachomatis clinical isolates identified as tetracycline resistant do not exhibit resistance in vitro: whole-genome sequencing reveals a mutation in porB but no evidence for tetracycline resistance genes. Microbiology. 2013;159:748-56.

102. Sandoz KM, Rockey DD. Antibiotic resistance in Chlamydiae. Future Microbiol. 2010;5:1427-42.

103. Yeruva L, Melnyk S, Spencer N, Bowlin A, Rank RG. Differential susceptibilities to azithromycin treatment of chlamydial infection in the gastrointestinal tract and cervix. Antimicrob Agents Chemother. 2013;57:6290-4. Report indicates that antibiotic levels sufficient to cure murine C. muridarum genital infection are ineffective against concomitant gastrointestinal infection. 